\title{
A Novel Linear Active Disturbance Rejection Control Design for Air-Breathing Supersonic Vehicle Attitude System with Prescribed Performance
}

\author{
Chao Ming $(\mathbb{D}$ and Xiaoming Wang \\ School of Mechanical Engineering, Nanjing University of Science and Technology, Nanjing 210094, China \\ Correspondence should be addressed to Chao Ming; nust802mc@126.com
}

Received 1 December 2019; Revised 5 June 2020; Accepted 13 June 2020; Published 1 August 2020

Academic Editor: Franco Bernelli-Zazzera

Copyright (c) 2020 Chao Ming and Xiaoming Wang. This is an open access article distributed under the Creative Commons Attribution License, which permits unrestricted use, distribution, and reproduction in any medium, provided the original work is properly cited.

\begin{abstract}
This paper investigates the design problem of the attitude controller for air-breathing supersonic vehicle subject to uncertainties and disturbances. Firstly, the longitudinal model is established for the attitude controller design which is devised as a strict feedback formulation, and a transformed tracking error is derived with the prescribed performance control technique such that it can limit the tracking error to a predefined region. Then, a novel linear active disturbance rejection control scheme is proposed for the attitude system to enhance the steady-state and transient-state performances by incorporating the transformed tracking error. On the basis of the Lyapunov stability theorem, the convergence and stability characteristics are both rigorously proved for the closed-loop system. Finally, extensive contrast simulations are conducted to demonstrate the effectiveness, robustness, and advantage of the proposed control strategy.
\end{abstract}

\section{Introduction}

Hypersonic technique is a wide development prospect in both military and civil fields $[1,2]$. As a derivative of hypersonic technique, air-breathing supersonic vehicle (ASV) is a new type of aircraft with large airspace, super flight velocity, long range, and high precision compared with the traditional aircraft [3], and flight guidance-control design is a key technology in the ASV system. However, as the special structure and changeable flight conditions, the vehicle dynamics are peculiar including the fast time varying, nonlinearity, uncertainty, and multiple disturbances [4], which lead to more difficulties and complexities in the control design and analysis. In addition, the ASV usually attacks the target at a supersonic velocity such that the flight states are changed rapidly which imposes a higher requirement for the transient characteristic of the control system [5]. Therefore, the control performance index such as the overshoot, steady-state error, convergence rate, and robustness must be considered the major designed indexes in the control system design of such flight vehicle.
In the past few years, the air-breathing vehicle technique has attracted the wide attention of domestic and foreign scientific research institutions and scholars due to the aforementioned superiorities, and various control methods have been explored for such vehicles, such as linear parameter varying (LPV) method [6], dynamic inversion $[7,8]$, trajectory linearization control $[9,10]$, fuzzy control method [11], dynamic surface control [12, 13], back-stepping control method [14, 15], neural network [16, 17], and sliding mode control [18-21].

It is worth noting that the above listed control method achieved an outstanding control effect for air-breathing vehicle, but most mainly fasten the attention on the stability, robustness, and accuracy of the control system. However, the situation of autodisturbance rejection is seldom considered in control design. As we all know that the active disturbance rejection control (ADRC) method can achieve a satisfactory performance for nonlinear systems, where the parameter perturbations and external disturbances can be estimated and rejected actively, it is successfully applied in industrial control $[22,23]$. However, there is an inevitable 
problem that many parameters are needed to be tuned which limits the application of ADRC in practice. In this connection, a linear active disturbance rejection control (LADRC) was firstly designed by Prof. Gao [24] which is extremely simple and easily implementable and it has been widely extended to various industrial control fields, such as electric erection system [25], hovercraft system [26], servo systems [27], wind power systems [28], and photobioreactor [29]. Meanwhile, $[30,31]$ adopt the LADRC approach to design the attitude control for a supersonic missile. As far as we know, there are few literatures which concern with the attitude control system design by adopting the LADRC method. Furthermore, in the design process of the above LADRC scheme, both did not consider the transient performance design. Therefore, it is vitally essential for the ASV attitude control system design that can guarantee the system for both the steady-state performance and transient performance with LADRC method.

Recently, a newly emerging control method called the prescribed performance control (PPC) for the nonlinear system was firstly proposed by $\mathrm{Na}$ et al. [32], where the tracking error can converge to an arbitrarily predefined small set and the convergence rate and maximum overshoot can be delimited less than a prespecified constant. Owing to the significant advantages in improving the control performance, the PPC is introduced to the vehicle suspension system [33], manipulator system [34], servo mechanisms [35, 36], unmanned aerial vehicle [37], spacecraft [38-40], etc., and several new ideas of the PPC design are emerged; [41] proposes a new error transformation method and the performance function so that the limitation of PPC on the known initial error can be relaxed. [42] investigates a new PPC methodology for the longitudinal dynamic model of an air-breathing hypersonic vehicle via neural approximation that the satisfactory transient performance with small overshoot can be achieved. [43] presents a new error transformation to reduce the complexity of the control system which is caused by conventional error constraint approaches. Berger [44] proposes a novel prescribed performance controller which can guarantee the output to stay within a prescribed performance funnel bound by incorporating the funnel control with the PPC technique. [45] designs a new prescribed performance controller without an approximation structure which can avoid the large amount of calculation and some specific problems of the fuzzy or neural network method in the approximation process. Although extensive achievements have been yielded in theory and application of the PPC technique, the previous controller design methods are mainly neural network control, backstepping control, dynamic surface control, and sliding model control. To our best knowledge, no results on the LADRC design with the PPC technique have been reported for nonlinear systems.

Motivated by the aforementioned discussions, this study investigates a novel LADRC scheme for the longitudinal attitude mode of the air-breathing supersonic vehicle in the presence of uncertain dynamics and external disturbances with a prescribed performance constraint. The main contributions of this paper are summarized as follows: (1) A novel LADRC approach design method with a prescribed perfor- mance constraint is firstly proposed for the ASV attitude system with multiple disturbances. (2) The proposed control scheme does not need the knowledge of the flight dynamic model, and the uncertainty and disturbance can be actively estimated and compensated into the control signal. (3) The proposed controller can improve the steady-state and transient performances of the ASV attitude system compared with the traditional LADRC method. (4) The system stability and convergence characteristic are both proved strictly.

The rest of this paper is outlined as follows. The considered ASV attitude control model is established and the prior knowledge and problem formulation is given in Section 2. Section 3 presents the LADRC-based prescribed performance attitude controller design procedures. Comparative simulation results are provided in Section 4. Finally, some conclusions of this work are presented in Section 5.

\section{Problem Statement and Preliminaries}

In the section, the considered ASV attitude dynamic model is presented; then, some basic definitions of the prescribed performance control method are provided for the subsequent analysis, and finally, the objective of controller design is outlined.

2.1. Vehicle Attitude Model. For the subsequent design, the nonlinear longitudinal model of the ASV attitude system in this paper is derived on the basis of the model which includes the altitude, velocity, and attitude motion of the hypersonic vehicle modeled in [46, 47]. In addition, the main purpose is to investigate a novel attitude control method of ASV, and the attitude dynamics belongs to a fast motion compared with the change of velocity and altitude. With design simplification and no loss of generality, the changes of altitude and velocity can be ignored due to the limited influence on attitude dynamics. Hence, the ASV attitude system can be simplified as follows:

$$
\left\{\begin{array}{l}
\dot{\vartheta}=\omega_{z}, \\
\dot{\omega}_{z}=\frac{M_{z}}{J_{z}}, \\
\alpha=\vartheta-\theta,
\end{array}\right.
$$

where the pitch moment $M_{z}$ is defined as

$$
M_{z}=\bar{q} S L\left(m_{0}+m_{z}^{\alpha} \alpha+m_{z}^{\alpha^{2}} \alpha^{2}\right)+\bar{q} S L m_{z}^{\delta_{z}} \delta_{z}
$$

where elevator deflection $\delta_{z}$ is the control input, and $m_{0}, m_{z}^{\alpha}$, $m_{z}^{\alpha^{2}}$, and $m_{z}^{\delta_{z}}$ are the aerodynamic coefficients.

For simplicity, defining the state $\left[x_{1}, x_{2}\right]=\left[\vartheta, \omega_{z}\right]$ and combining with (1) and (2), the ASV attitude control system can be described with the strict feedback state-space form:

$$
\left\{\begin{array}{l}
\dot{x}_{1}=x_{2}, \\
\dot{x}_{2}=f+b u,
\end{array}\right.
$$


where

$$
\left\{\begin{array}{l}
f=\frac{\bar{q} S L\left(m_{0}+m_{z}^{\alpha} \alpha+m_{z}^{\alpha^{2}} \alpha^{2}\right)}{J_{z}}+\Delta \\
b=\frac{\bar{q} S L m_{z}^{\delta_{z}}}{J_{z}} \\
u=\delta_{z}
\end{array}\right.
$$

Here, the term $f$ is described as "total disturbance," which consists of the unknown dynamic uncertainty and external disturbance.

Remark 1 . The uncertainty term $\Delta$ is mainly the result of parameter uncertainties and external disturbances, e.g., the aerodynamic parameters perturbations and wind interference.

Assumption 1. The total disturbance $f$ is differentiable, and its derivative $\dot{f}$ is described as $\dot{f}=h\left(x_{1}, x_{2}, \Delta\right)$. The disturbance $\Delta$ in system (3) is unknown, but the disturbance and its derivative are all bounded.

2.2. Prescribed Performance Theory. The prescribed performance denotes that the prescribed transient and steadystate performances can strictly limit the tracking error to a predesigned small residual set. Based on the prescribed performance concepts [31], the prescribed performance can be obtained in the condition that the tracking error $e(t)$ evolves in the predefined bounds with a decreasing smooth function as follows:

$$
-\rho(t)<e(t)<\rho(t), \forall t>0
$$

The prescribed performance function (PPF) $\rho(t)$ is selected as [38]

$$
\rho(t)=\left(\rho_{0}-\rho_{\infty}\right) \exp (-\kappa t)+\rho_{\infty},
$$

where $\rho_{0}>\rho_{\infty}>0$ and $\kappa>0$. The maximum overshoot, steady-state error, and convergence rate will be limited by selecting the appropriate parameters $\rho_{0}, \rho_{\infty}$, and $\kappa$, respectively. For a more intuitive insight of the concept, Figure 1 illustrates the schematic diagram of the aforementioned prescribed performance theory.

It is worth to mention that the complexity of the controller design will be significantly increased by directly adopting (5) and (6) which correspond to an additional constraint of the controlled system. In order to evade this problem, the system with constrains can be converted to an equivalent disengaged one by introducing an error transformation function $T(\cdot)$, i.e.,

$$
e(t)=\rho(t) T(\varepsilon(t))
$$

where $\varepsilon(t)$ is the transformed tracking error, and $T(\cdot)$ is smooth and strictly increasing which satisfies the conditions $-1<T(\varepsilon)<1$ and $\lim _{\varepsilon \rightarrow+\infty} T(\varepsilon)=1, \lim _{\varepsilon \rightarrow-\infty} T(\varepsilon)=-1$.

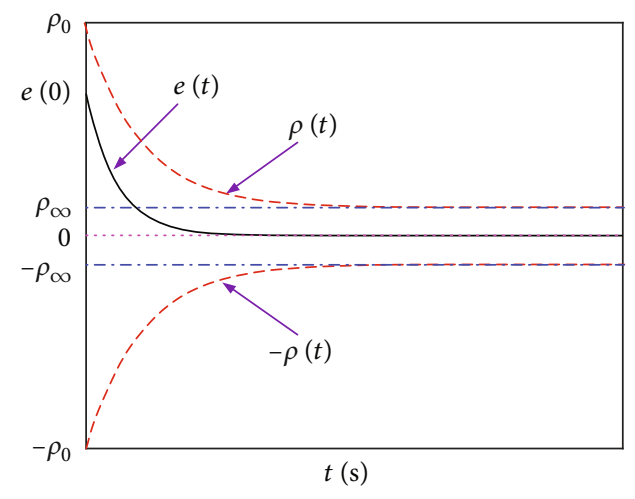

FIGURE 1: The prescribed performance illustration.

Here, we design the error transformation function $T(\cdot)$ as

$$
T(\varepsilon)=\frac{\exp (\varepsilon)-\exp (-\varepsilon)}{\exp (\varepsilon)+\exp (-\varepsilon)}
$$

According to the property of the function $T(\cdot)$, we can inversely derive the error $\varepsilon(t)$ as follows:

$$
\varepsilon(t)=T^{-1}(\lambda)=\frac{1}{2}\left[\ln \left(1+\lambda_{1}\right)-\ln \left(1-\lambda_{1}\right)\right]
$$

where $\lambda=e(t) / \rho(t)$ is the normalized tracking error.

Remark 2. In the subsequent parts, the transformed error $\varepsilon(t)$ will be used into the controller in place of the tracking error $e(t)$ to deal with the problem of control system design with prescribed performance constraint.

2.3. Control Objective. The control objective is the proposed novel controller $u$ for the ASV attitude system (3) that the state $x_{1}$ track the desired command $x_{1 c}$ accurately, and the tracking error $e_{1}=x_{1 \mathrm{c}}-x_{1}$ can be limited within a predefined bound with a satisfactory prescribed performance in spite of multiple disturbances including unmodeled dynamics, uncertainties, and external disturbances, i.e., the objective can be expressed as follows:

(1) The state $x_{1}$ can accurately track the desired command $x_{1 \mathrm{c}}$ with the unknown multiple disturbances

(2) The output tracking error $e_{1}$ is stabilized at the origin with a prescribed maximum overshoot, the steadystate error, and convergence rate

(3) The closed-loop system states are both stable and robust to the uncertainties and disturbances

\section{Control System Design and Stability Analysis}

In this part, a novel ASV attitude controller is proposed based on the LADRC method by introducing the PPC technique, which can improve the steady-state and transient performances of the ASV attitude control system. Then, the 


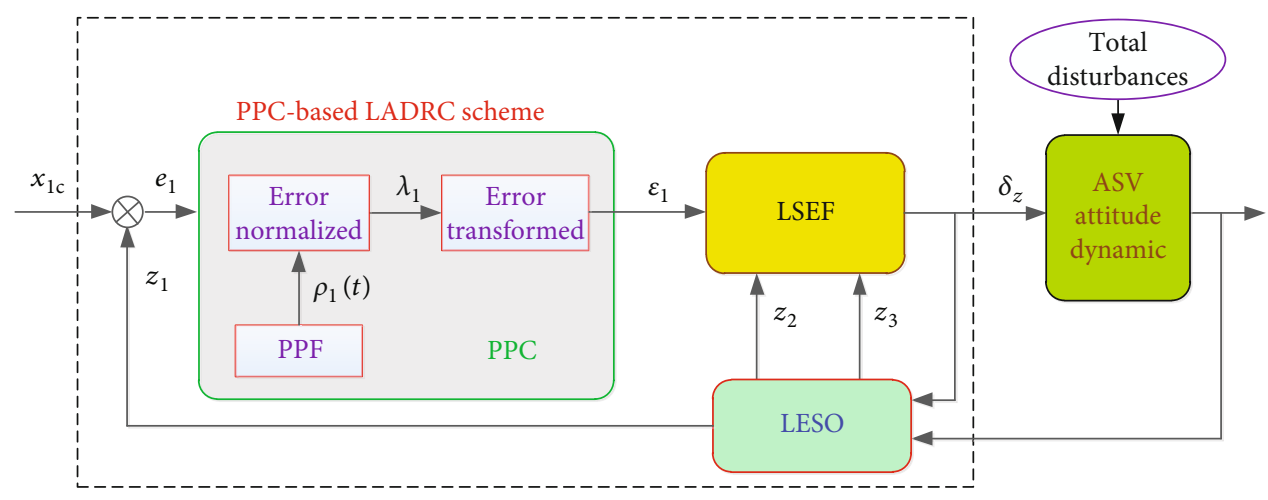

Figure 2: The block diagram of the presented controller.

convergence property and stability analysis for the control system are provided on the basic of the Lyapunov method.

3.1. Attitude Control System Design. The block diagram of the presented control system is depicted in Figure 2, which mainly consists of three parts: prescribed performance control (PPC), linear extended state observer (LESO), and linear state error feedback (LSEF).

In view of the previous description, the design process of the ASV attitude control system is illustrated as follows:

Step 1.

The LESO is the core part of the LADRC; it can generate the estimation of the states and the disturbances in real time; the estimated value can be used to compensate the disturbances to the controller which can enhance the robustness of the system. According to system (3), a three-order LESO is constructed as follows:

$$
\left\{\begin{array}{l}
\dot{z}_{1}=z_{2}+\beta_{1}\left(x_{1}-z_{1}\right), \\
\dot{z}_{2}=z_{3}+\beta_{2}\left(x_{1}-z_{1}\right)+b u \\
\dot{z}_{2}=\beta_{3}\left(x_{1}-z_{1}\right)
\end{array}\right.
$$

where $z_{1}, z_{2}$, and $z_{3}$ are the observer states, and $\beta_{1}, \beta_{2}$, and $\beta_{3}$ are the designed gains which can be chosen with the poleplacement method [24] as follows:

$$
\begin{aligned}
& \beta_{1}=3 \omega_{0} \\
& \beta_{2}=3 \omega_{0}^{2} \\
& \beta_{3}=\omega_{0}^{3}
\end{aligned}
$$

where $\omega_{0}$ is the observer bandwidth.

With the properly selected gains, $z_{1}, z_{2}$ will converge to the system states $x_{1}$ and $x_{2}$, respectively, and $z_{3}$ will accurately track the total disturbance term $f$, i.e., $z_{1} \longrightarrow x_{1}$, $z_{2} \longrightarrow x_{2}, z_{3} \longrightarrow f$.

Note that the LESO can estimate the states and total disturbance exactly without the mathematical precise model, it is only dependent of the system input and output information.

Step 2.

The PPC can transform the tracking error into an equivalent unconstrained one by incorporating the transformation function and the prescribed performance function such that the tracking error can be limited in the envelope of the prescribed performance bounds (PPB).

Here, we define the error $\tilde{e}_{1}$ as

$$
\tilde{e}_{1}=x_{1 \mathrm{c}}-z_{1}
$$

where $x_{1 \mathrm{c}}$ is the desired command and $z_{1}$ is the estimation value of $x_{1}$ obtained by LESO.

Subsequently, the normalized error $\lambda_{1}$ is given by

$$
\lambda_{1}=\frac{\tilde{e}_{1}}{\rho_{1}(t)},
$$

where $\rho_{1}(t)$ is a similar PPF defined in (6), and it is expressed as

$$
\rho_{1}(t)=\left(\rho_{10}-\rho_{1 \infty}\right) \exp \left(-\kappa_{1} t\right)+\rho_{1 \infty}
$$

where $\rho_{10}, \rho_{1 \infty}$, and $\kappa_{1}$ are all positive constants.

Then, the transformed error is obtained by

$$
\varepsilon_{1}=T^{-1}\left(\lambda_{1}\right)=\frac{1}{2}\left[\ln \left(1+\lambda_{1}\right)-\ln \left(1-\lambda_{1}\right)\right] .
$$

Step 3.

Based on the accurate estimation and the designed transformed error, the LSEF can approximately simplify the system to a disturbance-free form meanwhile reconciling the performance of the system. The final control law is design as follows:

$$
u=u_{0}+u_{f}
$$

where term $u_{0}$ denotes a linear state error feedback control term that guarantees the system is asymptotically stable, and term $u_{f}$ represents the dynamic compensation control term to suppress the unfavourable consequence of the total disturbance such that it can enhance the robustness of the ASV attitude control system.

Here, the control subitem $u_{0}$ adopted the linear proportional and derivative (PD) control framework by 
introducing the transformed error $\varepsilon_{1}$ of PPC which is designed as follows:

$$
u_{0}=\frac{k_{p} \varepsilon_{1}-k_{d} z_{2}}{b}
$$

where $k_{p}, k_{d}$ are the controller gains and $z_{2}$ is the estimation value of $x_{2}$ obtained by LESO, and expression of $b$ is given in (3).

Meanwhile, the control subitem $u_{f}$ compensates the disturbances with the estimated value $z_{3}$, which is given by

$$
u_{f}=-\frac{z_{3}}{b} .
$$

Thus, the controller $u$ is obtained in terms of (16), (17), and (18) as follows:

$$
u=\frac{k_{p} \varepsilon_{1}-k_{d} z_{2}-z_{3}}{b} .
$$

Note that the controller $u_{0}$ introduces the transformed error $\varepsilon_{1}$ instead of $\tilde{e}_{1}$ which can enhance the transient and steadystate performances with the PPC technique.

3.2. Stability Analysis. In this subsection, the observer convergence and the closed-loop system stability will be analysed with the listed theorem. Prior to investigating, the following lemmas are introduced.

Lemma 1. For system $\dot{\boldsymbol{\eta}}(t)=N \mathbf{\eta}(t)+\mathbf{g}(t)$, where $N$ is a $n \times n$ matrix, and $\boldsymbol{\eta}(t)=\left[\eta_{1}(t), \eta_{2}(t), \cdots, \eta_{n}(t)\right], \quad \mathbf{g}(t)=\left[g_{1}(t)\right.$, $\left.g_{2}(t), \cdots, g_{n}(t)\right]$, if $\lim _{t \rightarrow \infty}\|\mathbf{g}(t)\|=0$ and $N$ is Hurwitz, then $\lim _{t \rightarrow \infty}\|\mathbf{\eta}(t)\|=0$ holds.

This lemma has been proofed in detail which can be seen in [48].

Lemma 2. If the transformed error $\varepsilon(t)$ can be controlled to be bounded, i.e., the condition $|\varepsilon(t)| \leq \varepsilon_{M}$ holds where $\varepsilon_{M}>0$ is constant; then, the tracking error $e(t)$ can be controlled within a prescribed boundary, i.e., $-\rho(t)<e(t)<\rho(t), \forall t>0$ holds.

This lemma has been proofed in detail which can be seen in $[38]$.

Theorem 1. For system (3) and LESO (10), if $\dot{f}=h\left(x_{1}, x_{2}, \Delta\right)$ satisfies the Lipschitz condition in the definition domain, there exist a constant $\omega_{0}>0$ such that the estimated states $z_{1}, z_{2}$, and $z_{3}$ can converge to the state $x_{1}, x_{2}$, and $f$, respectively, i.e., the observer errors satisfy that $\lim _{t \rightarrow \infty} e_{z i}(t)=0, i=1,2,3$, where the observer errors are defined as $e_{z 1}=x_{1}-z_{1}, e_{z 2}=x_{2}$ $-z_{2}$, and $e_{z 3}=f-z_{3}$.
Proof. Define the observer errors as

$$
\begin{aligned}
& e_{z 1}=x_{1}-z_{1}, \\
& e_{z 2}=x_{2}-z_{2}, \\
& e_{z 3}=f-z_{3} .
\end{aligned}
$$

From (3) and (10), the observer error dynamic can be given by

$$
\left\{\begin{array}{l}
\dot{e}_{z 1}=-3 \omega_{0} e_{z 1}+e_{z 2} \\
\dot{e}_{z 2}=-3 \omega_{0}^{2} e_{z 1}+e_{z 3} \\
\dot{e}_{z 3}=-\omega_{0}^{3} e_{z 1}+\dot{f}
\end{array}\right.
$$

Let $\tilde{\mathbf{e}}=\left[\tilde{e}_{1}, \tilde{e}_{2}, \tilde{e}_{3}\right]=\left[e_{z 1}, e_{z 2} / \omega_{0}, e_{z 3} / \omega_{0}{ }^{2}\right]$ and $\dot{f}=h\left(x_{1}\right.$, $\left.x_{2}, \Delta\right)$, then $(21)$ can be rewritten as follows:

$$
\dot{\tilde{\mathbf{e}}}=\omega_{0} \tilde{\mathbf{A}} \tilde{\mathbf{e}}+\tilde{\mathbf{B}} \frac{h\left(x_{1}, x_{2}, \Delta\right)}{\omega_{0}{ }^{3}},
$$

where $\tilde{\mathbf{A}}=\left[\begin{array}{lll}-3 & 1 & 0 \\ -3 & 0 & 0 \\ -1 & 0 & 0\end{array}\right], \tilde{\mathbf{B}}=\left[\begin{array}{l}0 \\ 0 \\ 1\end{array}\right]$.

As Ãsatisfies the Hurwitz stability, the condition $\mathbf{P} \tilde{\mathbf{A}}+$ $\tilde{\mathbf{A}}^{\mathrm{T}} \mathbf{P}=-\mathbf{I}$ holds, where the matrix $\mathbf{I}$ is 3-order identity matrix and $\mathbf{P}$ is a positive definite Hermitian matrix and is expressed as

$$
\mathbf{P}=\left[\begin{array}{ccc}
1 & -\frac{1}{2} & -1 \\
-\frac{1}{2} & 1 & -\frac{1}{2} \\
-1 & -\frac{1}{2} & 4
\end{array}\right]
$$

Design the following Lyapunov function candidate

$$
V_{1}=\tilde{\mathbf{e}}^{\mathrm{T}} \mathbf{P} \tilde{\mathbf{e}} .
$$

Substituting (23) into (24) yields

$$
V_{1}=\tilde{e}_{1}^{2}+\tilde{e}_{2}^{2}+4 \tilde{e}_{3}^{2}-\tilde{e}_{1} \tilde{e}_{2}-2 \tilde{e}_{1} \tilde{e}_{3}-\tilde{e}_{2} \tilde{e}_{3} .
$$

The time derivative of $V_{1}$ along (25) is

$$
\begin{aligned}
\dot{V}_{1}= & 2 \tilde{e}_{1} \dot{\tilde{e}}_{1}+2 \tilde{e}_{2} \dot{\tilde{e}}_{2}+8 \tilde{e}_{3} \dot{\tilde{e}}_{3}-\dot{\tilde{e}}_{1} \tilde{e}_{2}-\tilde{e}_{1} \dot{\tilde{e}}_{2} \\
& -2 \dot{\tilde{e}}_{1} \tilde{e}_{3}-2 \tilde{e}_{1} \dot{\tilde{e}}_{3}-\dot{\tilde{e}}_{2} \tilde{e}_{3}-\tilde{e}_{2} \dot{\tilde{e}}_{3}, \\
= & -\omega_{0}\left(\tilde{e}_{1}^{2}+\tilde{e}_{2}^{2}+\tilde{e}_{3}^{2}\right)+\left(-2 \tilde{e}_{1}-\tilde{e}_{2}+8 \tilde{e}_{3}\right) \frac{h\left(x_{1}, x_{2}, \Delta\right)}{\omega_{0}^{2}} \\
\leq & -\omega_{0}\|\tilde{\mathbf{e}}\|^{2}+\left(-2 \tilde{e}_{1}-\tilde{e}_{2}+8 \tilde{e}_{3}\right) \frac{\left|h\left(x_{1}, x_{2}, \Delta\right)\right|}{\omega_{0}^{2}} .
\end{aligned}
$$


The term $h\left(x_{1}, x_{2}, \Delta\right)$ satisfies [49].

$$
\left|h\left(x_{1}, x_{2}, \Delta\right)\right| \leq k\|\tilde{\mathbf{e}}\|,
$$

where $k>0$ is constant.

Meanwhile, as the equation $-2 \tilde{e}_{1}-\tilde{e}_{2}+8 \tilde{e}_{3}=2 \tilde{\mathbf{e}}^{\mathrm{T}} \mathbf{P} \tilde{\mathbf{B}}$ holds, we can obtain that

$$
\left(-2 \tilde{e}_{1}-\tilde{e}_{2}+8 \tilde{e}_{3}\right) \frac{\left|h\left(x_{1}, x_{2}, \Delta\right)\right|}{\omega_{0}^{2}} \leq 2 \tilde{\mathbf{e}}^{\mathrm{T}} \mathbf{P} \tilde{\mathbf{B}} k \frac{\|\tilde{\mathbf{e}}\|}{\omega_{0}^{2}}
$$

In addition, the following inequality holds

$$
\|\mathbf{P} \tilde{\mathbf{B}} k\|^{2}-2 \mathbf{P} \tilde{\mathbf{B}} k+1 \geq 0 .
$$

Thus, we obtain

$$
2 \tilde{\mathbf{e}}^{\mathrm{T}} \mathbf{P} \tilde{\mathbf{B}} k \frac{\|\tilde{\mathbf{e}}\|}{\omega_{0}{ }^{2}} \leq K\|\tilde{\mathbf{e}}\|^{2},
$$

where $K=\left(\left\|\mathbf{P B}_{\mathrm{Z}} k\right\|^{2}+1\right) / \omega_{0}{ }^{2}$.

Based on (26), (28), and (30), we can obtain that

$$
\dot{V}_{1} \leq-\left(\omega_{0}-K\right)\left\|\tilde{\mathbf{e}}_{\mathbf{Z}}\right\|^{2} .
$$

That is, if $\omega_{0}>K$ such that $\dot{V}_{1}<0$. Therefore, $\lim _{t \rightarrow \infty} e_{z i}(t)=$ $0, i=1,2,3$ can be achieved, i.e., the estimation errors of the LESO (10) are asymptotically stable. The proof of Theorem 1 is completed.

Theorem 2. For system (3), LESO (10), and the proposed controller (19) with the prescribed performance function (14), if $\dot{f}=h\left(x_{1}, x_{2}, \Delta\right)$ satisfies the Lipschitz condition in definition domain, there exists constants $\omega_{0}>0$ and $\omega_{c}>0$ that can guarantee the system (3) to be asymptotically stable. Furthermore, the tracking error $e_{1}$ can be maintained in a predefined set, i.e., the inequality $-\rho_{1}(t)<e_{1}(t)<\rho_{1}(t)$ holds.

Proof. Define the tracking error of system (3) as

$$
\begin{aligned}
& e_{1}=x_{1 c}-x_{1}, \\
& e_{2}=x_{2 c}-x_{2} .
\end{aligned}
$$

As the control command $x_{1 \mathrm{c}}$ is assumed as a constant, we have $x_{2 \mathrm{c}}=\dot{x}_{1 \mathrm{c}}=0$. The time derivative of $e_{1}$ and $e_{2} \operatorname{along}(3)$ is

$$
\begin{aligned}
& \dot{e}_{1}=\dot{x}_{1 \mathrm{c}}-\dot{x}_{1}=-x_{2}=e_{2}, \\
& \dot{e}_{2}=\dot{x}_{2 c}-\dot{x}_{2}=-\dot{x}_{2}=-f-b u .
\end{aligned}
$$

Based on the expression of the transformed error $\varepsilon_{1}$, it yields

$$
\varepsilon_{1}=\frac{1}{2}\left[\ln \left(1+\lambda_{1}\right)-\ln \left(1-\lambda_{1}\right)\right]
$$

Then, the function $\ln \left(1+\lambda_{1}\right)$ and $\ln \left(1+\lambda_{1}\right)$ can be derived using Taylor's expansion as follows:

$$
\begin{aligned}
& \ln \left(1+\lambda_{1}\right)=\lambda_{1}-\frac{1}{2} \lambda_{1}^{2}+R_{2}\left(\lambda_{1}\right), \\
& \ln \left(1-\lambda_{1}\right)=-\lambda_{1}+\frac{1}{2} \lambda_{1}^{2}+R_{2}\left(\lambda_{1}\right),
\end{aligned}
$$

where $R_{2}\left(\lambda_{1}\right)$ is the 2 -order Taylor remainder. As the error $\lambda_{1}$ is relatively small near zero, thus the $R_{2}\left(\lambda_{1}\right)$ can be neglected.

Substituting (36) into (35), the transformed error $\varepsilon_{1}$ can be approximately expressed as

$$
\varepsilon_{1}=\lambda_{1}=\frac{x_{1 \mathrm{c}}-z_{1}}{\rho_{1}(t)}
$$

Substituting (38) into (19), the proposed controller (18) can be rewritten as

$$
u=\frac{k_{p}\left(x_{1 \mathrm{c}}-z_{1} / \rho_{1}(t)\right)-k_{d} z_{2}-z_{3}}{b}
$$

Substituting (39) into (34) yields

$$
\dot{e}_{2}=-f-b u=-k_{p} \frac{x_{1 \mathrm{c}}-z_{1}}{\rho(t)}+k_{d} z_{2}+z_{3}-f \text {. }
$$

Combining the expressions of the observer error and system error, we can obtain that

$$
\begin{aligned}
x_{1 \mathrm{c}}-z_{1} & =e_{1}+e_{z 1}, \\
z_{2} & =-\left(e_{2}+e_{z 2}\right), \\
z_{3}-f & =-e_{z 3} .
\end{aligned}
$$

Substituting (41) into (40) yields

$$
\dot{e}_{2}=-\frac{k_{p}}{\rho(t)}\left(e_{1}+e_{z 1}\right)-k_{d}\left(e_{2}+e_{z 2}\right)-e_{z 3} .
$$

From (33) and (44), the tracking error model is given by

$$
\dot{\mathbf{e}}=\mathbf{A}_{\mathbf{e}} \mathbf{e}+\mathbf{B}_{\mathbf{e}} \mathbf{e}_{z},
$$

where $\mathbf{e}=\left[e_{1}, e_{2}\right]^{\mathrm{T}}$ and $\mathbf{e}_{z}=\left[e_{z 1}, e_{z 2}\right]^{\mathrm{T}}$ are the system tracking error vector and the observer error vector, respectively. The matrix $\mathbf{A}_{\mathbf{e}}$ and $\mathbf{B}_{\mathbf{e}}$ are described as

$$
\begin{aligned}
\mathbf{A}_{\mathbf{e}} & =\left[\begin{array}{cc}
0 & 1 \\
-\frac{k_{p}}{\rho_{1}(t)} & -k_{d}
\end{array}\right], \\
\mathbf{B}_{\mathbf{e}} & =\left[\begin{array}{ccc}
0 & 0 & 0 \\
-\frac{k_{p}}{\rho_{1}(t)} & -k_{d} & -1
\end{array}\right] .
\end{aligned}
$$


Meanwhile, the prescribed performance function $\rho_{1}(t)$ satisfies

$$
\lim _{t \rightarrow \infty} \rho_{1}(t)=\lim _{t \rightarrow \infty}\left[\left(\rho_{10}-\rho_{1 \infty}\right) \exp \left(-\kappa_{1} t\right)+\rho_{1 \infty}\right]=\rho_{1 \infty} .
$$

Thus, under the condition $t \longrightarrow \infty$, the matrix $\mathbf{A}_{\mathbf{e}}$ and $\mathbf{B}_{\mathbf{e}}$ can be given as

$$
\begin{aligned}
& \mathbf{A}_{\mathbf{e}}=\left[\begin{array}{cc}
0 & 1 \\
-\frac{k_{p}}{\rho_{100}} & -k_{d}
\end{array}\right], \\
& \mathbf{B}_{\mathbf{e}}=\left[\begin{array}{ccc}
0 & 0 & 0 \\
-\frac{k_{p}}{\rho_{10}} & -k_{d} & -1
\end{array}\right] .
\end{aligned}
$$

According to Theorem 1, we can obtain that $\lim _{t \rightarrow \infty}\left\|\mathbf{e}_{z}\right\|=0$. Furthermore, if the characteristic polynomial $s^{2}+k_{d} s+k_{p} l$ $\rho_{100}$ satisfies the Routh criterion, here, we select $s^{2}+k_{d} s+$ $k_{p} / \rho_{1 \infty}=\left(s+\omega_{c}\right)^{2}$, where $\omega_{c}>0$ is the controller bandwidth, i.e., $k_{p}=\rho_{\infty} \omega_{c}^{2}, k_{d}=2 \omega_{c}$ such that the matrix $\mathbf{A}_{\mathbf{e}}$ is Hurwitz. Based on Lemma 1, we can obtain that the $\lim _{t \rightarrow \infty} e_{i}(t)=0, i=$ 1,2 holds, i.e., the system (3) is asymptotically stable.

Besides that, we can obtain the following equation by conducting the inverse operation based on the transformed error (15) as

$$
\exp \left(2 \varepsilon_{1}\right)=\frac{1+\lambda_{1}}{1-\lambda_{1}}
$$

Based on Lemma 2, if the transformed errors $\varepsilon_{1}=T^{-1}$ ( $\left.\lambda_{1}\right)$ can be controlled to be bounded, i.e., $\left|\varepsilon_{1}\right| \leq \varepsilon_{M 1}$ holds for positive constants $\varepsilon_{M 1}>0$. This further implies

$$
-1<\frac{\exp \left(-\varepsilon_{M 1}\right)-1}{\exp \left(-\varepsilon_{M 1}\right)+1} \leq \lambda_{1} \leq \frac{\exp \left(\varepsilon_{M 1}\right)-1}{\exp \left(\varepsilon_{M 1}\right)+1}<1 .
$$

From the fact $\lambda_{1}=e_{1}(t) / \rho_{1}(t)$ and $\rho_{1}(t)>0$, the error $e_{1}$ can be maintained within a predefined set, $-\rho_{1}(t)<e_{1}(t)<$ $\rho_{1}(t)$ holds. This completes the proof.

\section{Simulation Results and Analysis}

In order to evaluate the performance of the proposed ASV attitude controller, three numerical simulation cases are conducted in different configurations and scenarios.

4.1. Contrast Scheme. In order to demonstrate effectiveness and superiority of the proposed controller with the prescribed performance control-based LADRC (LADRC-PPC) method, the LADRC method [30] is introduced to the simu- lation scenarios for comparison study, and the controller is devised as follows:

$$
u_{\mathrm{LADC}}=\frac{k_{p}\left(x_{1 \mathrm{c}}-z_{1}\right)-k_{d} z_{2}-z_{3}}{b},
$$

where $z_{1}, z_{2}$, and $z_{3}$ is obtained by LESO.

4.2. Flight Conditions. The vehicle is assumed to maintain altitude $H=12 \mathrm{~km}$ cruise at constant velocity $V=1000 \mathrm{~m} / \mathrm{s}$ such that the flight path angle is $\theta=0$ deg, i.e., the variation of the angle of attack $\alpha$ is the same as that of the pitch angle $\vartheta$ based on the system (1). The aerodynamic coefficients are $m_{0}=0.0005, \quad m_{z}^{\alpha}=-0.018 \mathrm{deg}^{-1}, m_{z}^{\alpha^{2}}=-0.003 \mathrm{deg}^{-2}$, and $m_{z}^{\delta_{z}}=-0.035 \mathrm{deg}^{-1}$. The initial conditions for the ASV are set as $\vartheta(0)=0 \mathrm{deg}, \omega_{z}(0)=0 \mathrm{deg} / \mathrm{s}$.

4.3. Controller Parameters. The relevant parameters of PPF is designed as $\rho_{10}=1.5, \rho_{100}=0.05$, and $\kappa_{1}=5$, i.e., the PPF is selected as $\rho_{1}(t)=(1.5-0.05) \exp (-5 t)+0.05$. The observer bandwidth $\omega_{0}$ is set as $\omega_{0}=40$, and controller bandwidth $\omega_{c}$ is designed as $\omega_{c}=10$. Then, the observer gains $\beta_{1}, \beta_{2}$, and $\beta_{3}$ and controller gains $k_{p}, k_{d}$ can be obtained according to the correspondence with the bandwidth $\omega_{0}$ and $\omega_{\mathrm{c}}$.

4.4. Evaluation Index. For quantitatively contrasting the performance, the following performance indexes are introduced to evaluate the above control schemes.

(1) Convergence time (CT) $t_{\mathrm{c}}$ of the tracking error (TE) The convergence time $t_{\mathrm{c}}$ is assumed as the corresponding time that the tracking error $e_{1}$ is $e_{1} \leq 10^{-6}$ and continues at least 10 sampling periods.

(2) Average value (AV) $\mu_{\mathrm{e}}$ of the TE

$$
\mu_{\mathrm{e}}=\frac{1}{n} \sum_{i=1}^{n}\left|e_{1}(i)\right|
$$

where $n$ is the sample point number

(3) Standard deviation (SD) $\sigma_{\mathrm{e}}$ of the TE

$$
\sigma_{\mathrm{e}}=\sqrt{\frac{1}{n} \sum_{i=1}^{n}\left[\left|e_{1}(i)\right|-\mu_{\mathrm{e}}\right]^{2}}
$$

(4) Amount of control consumption (ACC) Q

$$
Q=\int_{t_{0}}^{t_{f}} u^{2} \mathrm{~d} t
$$

where $t_{0}$ and $t_{f}$ are the start time and end time, respectively, $u$ is the control input. 
TABLE 1: The comparative simulation results of Case 1.

\begin{tabular}{lccc}
\hline Index & LADRC & LADRC-PPC & Ratio \\
\hline CT $t_{\mathrm{c}}$ & $2.65 \mathrm{~s}$ & $0.99 \mathrm{~s}$ & $2.57: 1$ \\
$\mathrm{AV} \mu_{\mathrm{e}}$ & $0.07 \mathrm{deg}$ & $0.03 \mathrm{deg}$ & $2.33: 1$ \\
$\mathrm{SD} \sigma_{\mathrm{e}}$ & $0.18 \mathrm{deg}$ & $0.14 \mathrm{deg}$ & $1.28: 1$ \\
$\mathrm{ACC} Q$ & 1.26 & 1.30 & $1: 1.03$ \\
\hline
\end{tabular}

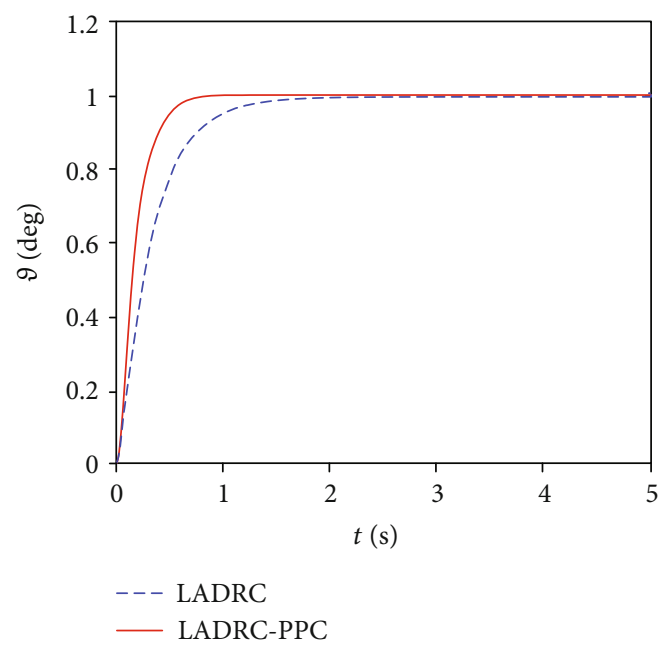

Figure 3: Pitch angle.

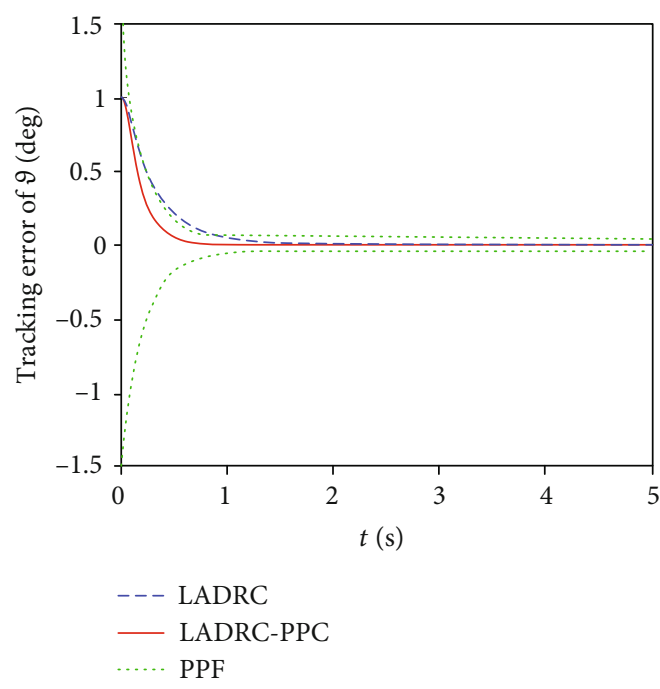

FIgURE 4: Tracking error of pitch angle.

Case 1. This simulation is conducted in a standard condition. The aerodynamic coefficients are set to be the nominal values without external disturbance, i.e., the term $\Delta$ in system (3) is $\Delta=0$.

The comparative results are summarized in Table 1 and Figures 3-10.

As depicted in Figures 3 and 4, the ASV can track the desired attitude command with the listed control approaches

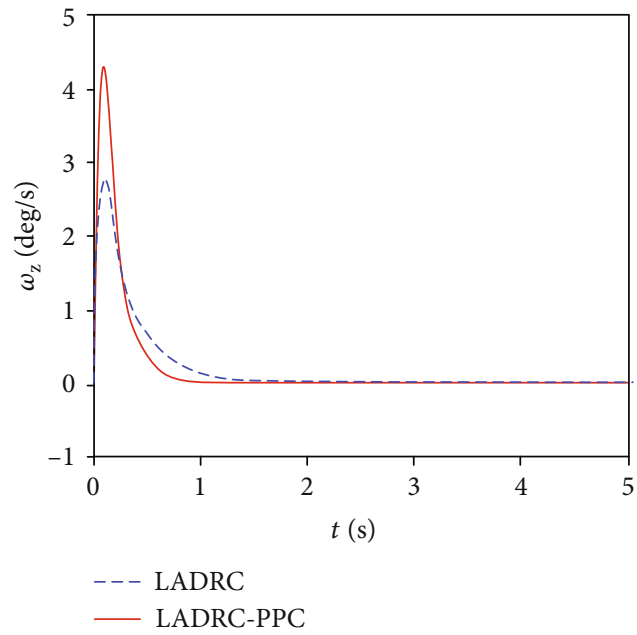

FIgURE 5: Pitch angular rate.

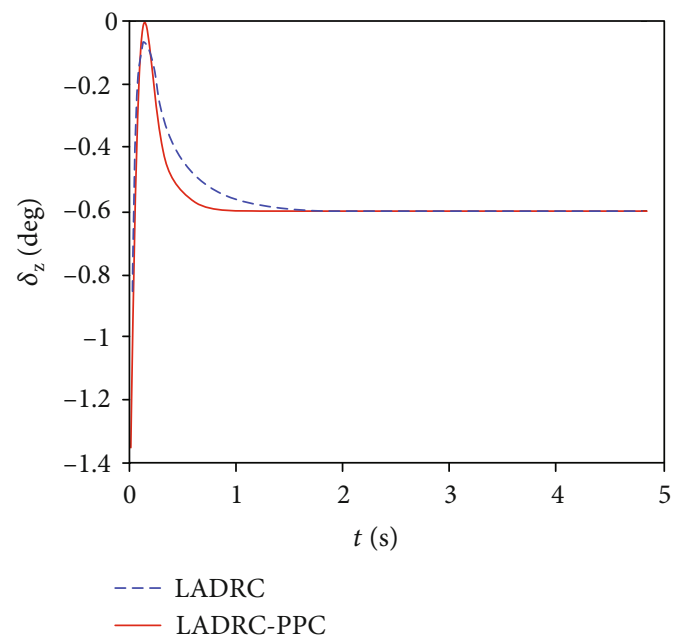

Figure 6: Control input signal-elevator deflection.

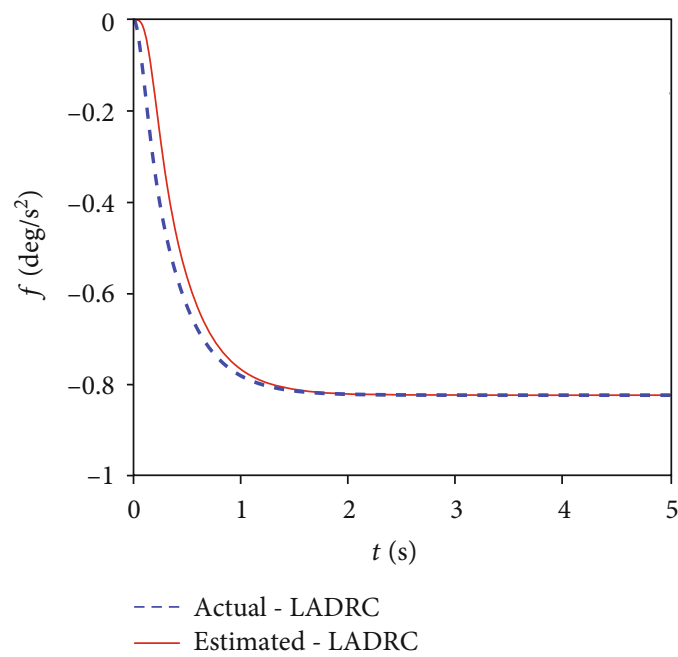

FIgURE 7: Estimation of $f$ with LADRC. 


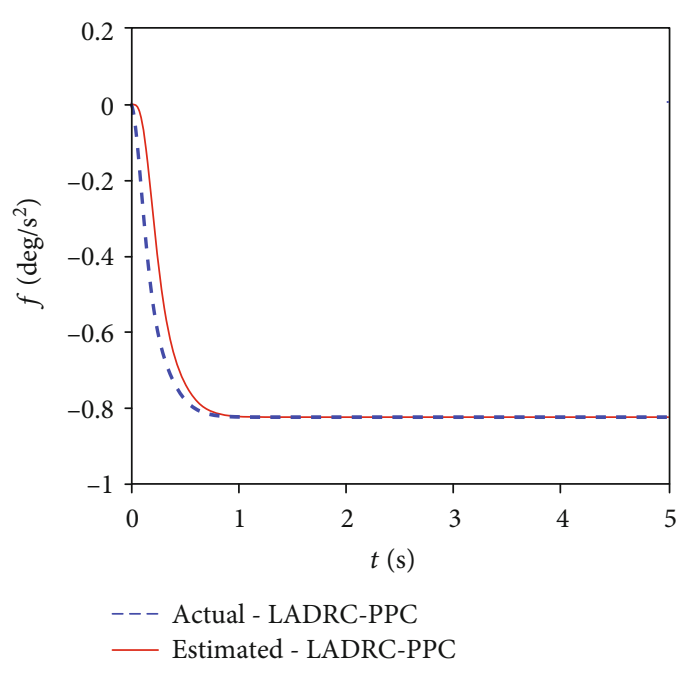

Figure 8: Estimation of $f$ with LADRC-PPC.

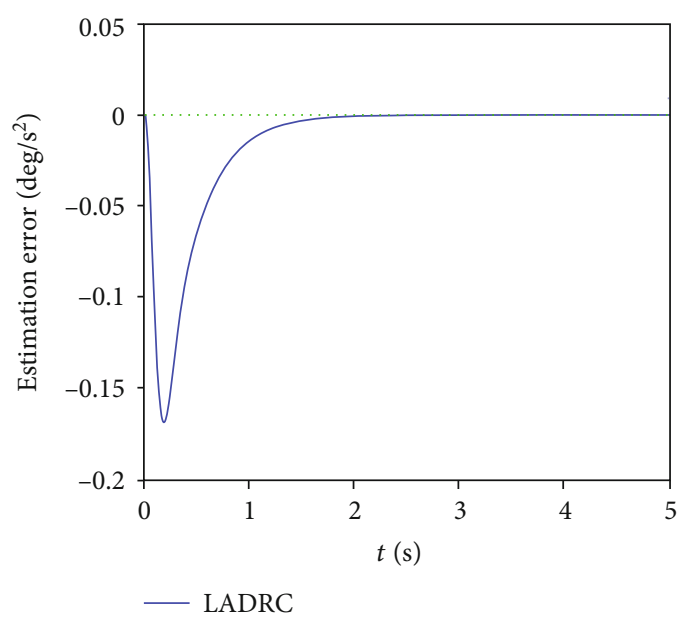

FIGURE 9: Estimation error of $f$ with LADRC.

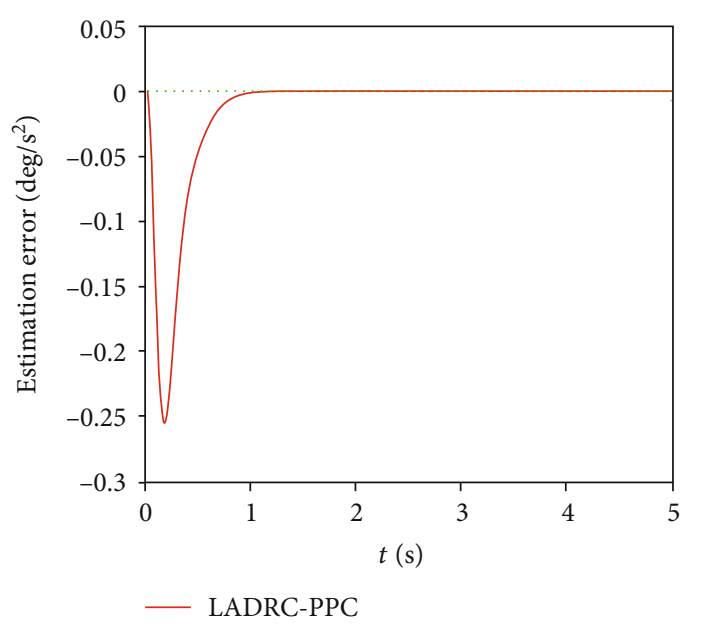

FIgURE 10: Estimation error of $f$ with LADRC-PPC. accurately. It is worth noting that the tracking error is limited in a prescribed set with a fairly satisfactory tracking error response by utilizing the presented LADRC-PPC controller. As listed in Table 1, the tracking error remains remarkably small with a faster rate and a much smaller average error which the average error is $0.03 \mathrm{deg}$, and converges to the neighbourhood of zero in approximately $0.99 \mathrm{~s}$ with the LADRC-PPC controller. However, the corresponding values with the LADRC scheme are $0.07 \mathrm{deg}$ and $2.55 \mathrm{~s}$, respectively. That is, the steady-state and transient performances of the ASV attitude system under the designed LADRC-PPC controller is obviously superior than the contrast LADRC which is owing to the help of introducing the PPC technology. We can see that initial value with the LADRC-PPC controller is slightly larger than the one with LADRC which is due to the faster convergence in the initial phase with the LADRCPPC approach as shown in Figures 5 and 6, and the amount of control consumption under the two schemes is virtually identical, i.e., the novel proposed LADRC-PPC scheme can achieve an excellent control performance with approximately the same control consumption in comparison with the LADRC method. Figures 7 and 8 illustrate the estimations of disturbances; it can be seen that LESO can precisely and rapidly estimate the total disturbances of the system, and the convergence rate of LESO under the LADRC-PPC is faster than that of LADRC as show in Figures 9 and 10.

Case 2. This case is performed in a perturbed condition to verify the robustness of the proposed method. The uncertainty term $\Delta$ is mainly considered two parts: (1) the model uncertainties $\Delta_{1}$, and the aerodynamic coefficients are presumed to decrease by $10 \%$ on the basis of standard values, the density of air $\rho$ is perturbed to be $+5 \%$ and (2) external disturbance $\Delta_{2}$, and it is considered an abrupt one $\Delta_{2}=0.1$ $\mathrm{deg} / \mathrm{s}^{2}(t \geq 3)$, i.e., the uncertainty term $\Delta$ is set as follows

$$
\Delta=\left\{\begin{array}{ll}
\Delta_{1} & (t<3) \\
\Delta_{1}+0.1 & (t \geq 3)
\end{array} \quad \mathrm{deg} / \mathrm{s}^{2} .\right.
$$

The comparative results are summarized in Table 2 and Figures 11-18.

From Figure 11, the ASV attitude system can track the desired command successfully by using the mentioned two control approaches in the presence of unknown dynamics, uncertainties, and disturbances. The tracking error response of the system with the proposed LADRC-PPC scheme is also better than the classic LADRC method in Figure 12. Especially, when the abrupt external disturbance occurs, the tracking error under the LADRC-PPC controller can converge to a compact set less than $0.5 \mathrm{~s}$, and the corresponding convergence time under the LADRC approach is nearly $2.0 \mathrm{~s}$. That is, the transient performance under the LADRC-PPC controller is significantly improved than the contrast LADRC in spite of unknown uncertainties and disturbances. From Figures 13 and 14, it can be seen that the variations of the states under the perturbed condition are generally accordant with the standard condition. Meanwhile, the amount of control consumption under the two controller is similar. In 
TABle 2: The comparative simulation results of Case 2.

\begin{tabular}{lccc}
\hline Index & LADRC & LADRC-PPC & Ratio \\
\hline CT $t_{\mathrm{c}}$ & $2.74 \mathrm{~s}$ & $1.10 \mathrm{~s}$ & $2.49: 1$ \\
$\mathrm{AV} \mu_{\mathrm{e}}$ & $0.07 \mathrm{deg}$ & $0.03 \mathrm{deg}$ & $2.33: 1$ \\
$\mathrm{SD} \sigma_{\mathrm{e}}$ & $0.18 \mathrm{deg}$ & $0.14 \mathrm{deg}$ & $1.28: 1$ \\
$\mathrm{ACC} Q$ & 1.06 & 1.10 & $1: 1.04$ \\
\hline
\end{tabular}

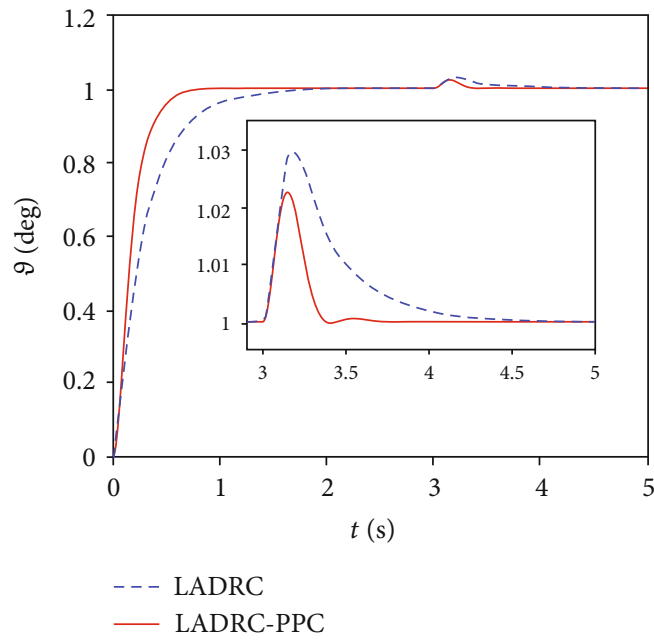

Figure 11: Pitch angle.

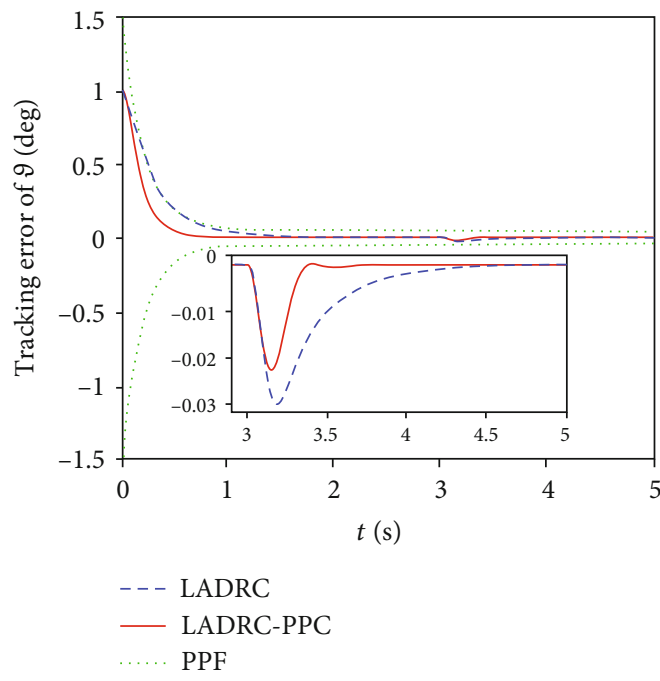

FIGURE 12: Tracking error of pitch angle.

addition, the two controllers can achieve an excellent tracking performance owing to the total disturbances can be precisely estimated with the LESO as shown in Figures 15-18.

Case 3. For further evaluating the robust performance of the proposed control scheme, a Monte Carlo analysis consisting

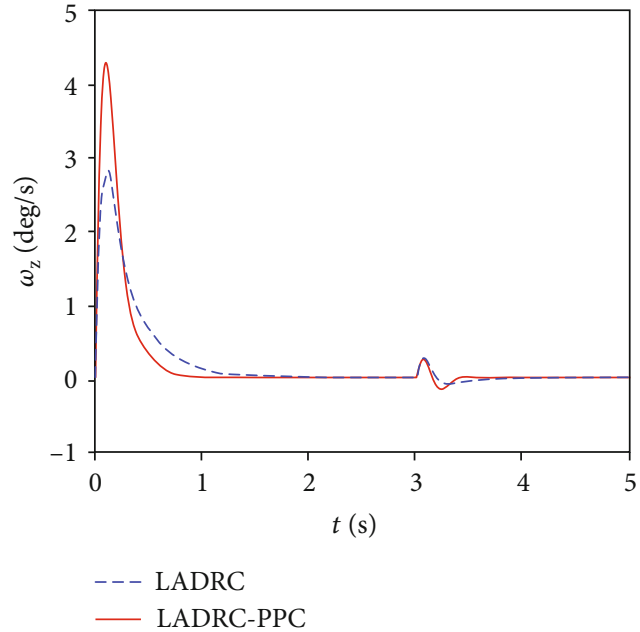

Figure 13: Pitch angular rate.

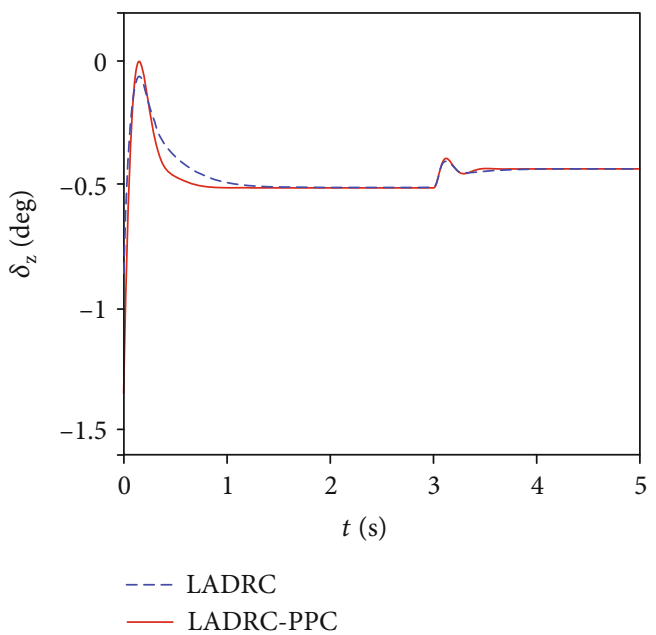

Figure 14: Control input signal-elevator deflection.

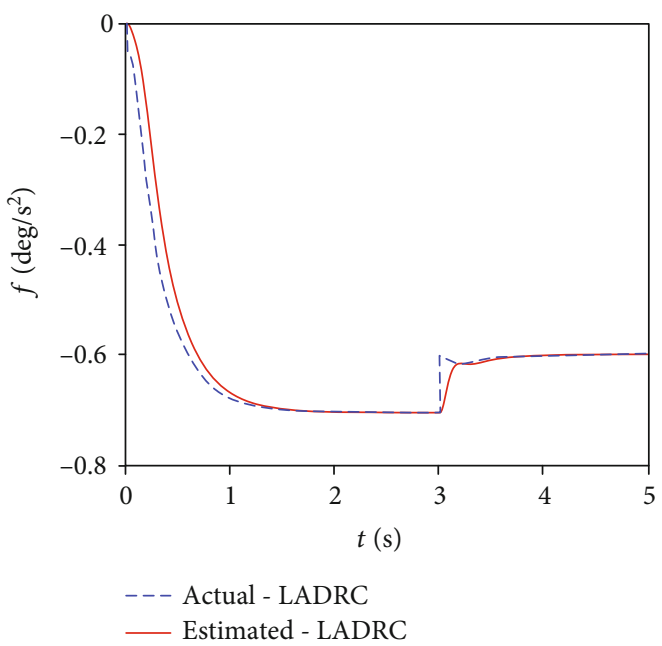

FIGURE 15: Estimation of $f$ with LADRC. 


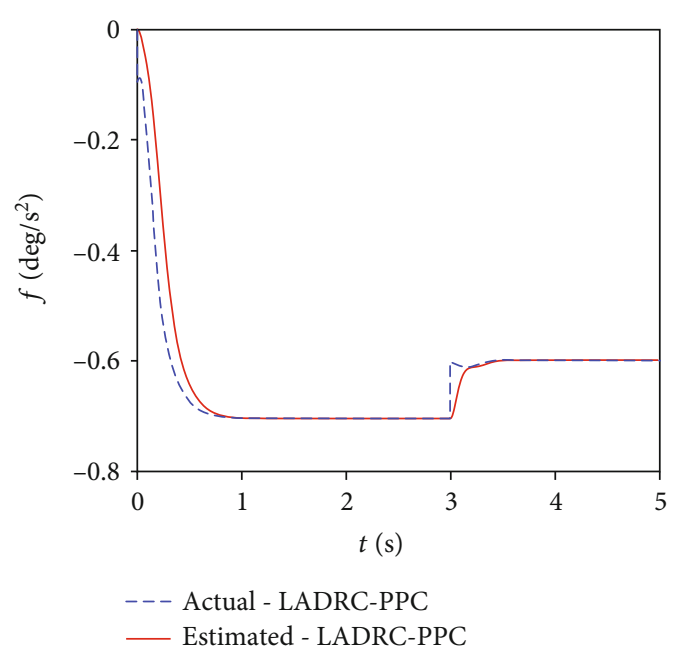

FIGURE 16: Estimation of $f$ with LADRC-PPC.

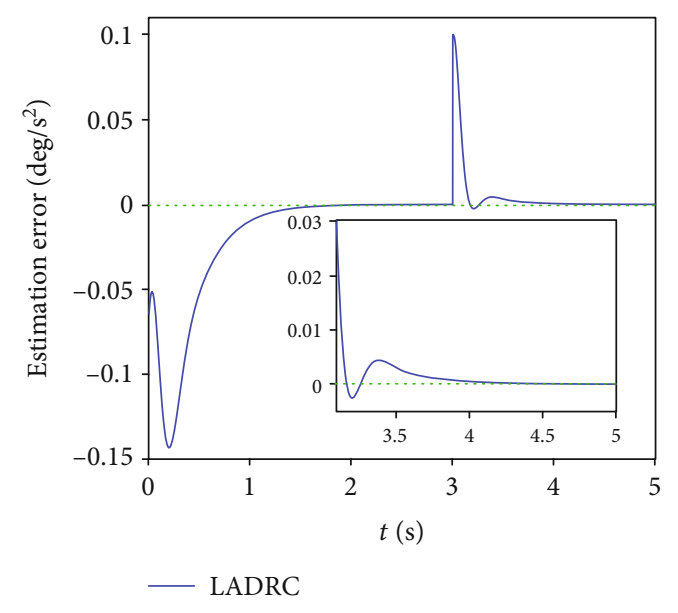

Figure 17: Estimation error of $f$ with LADRC.

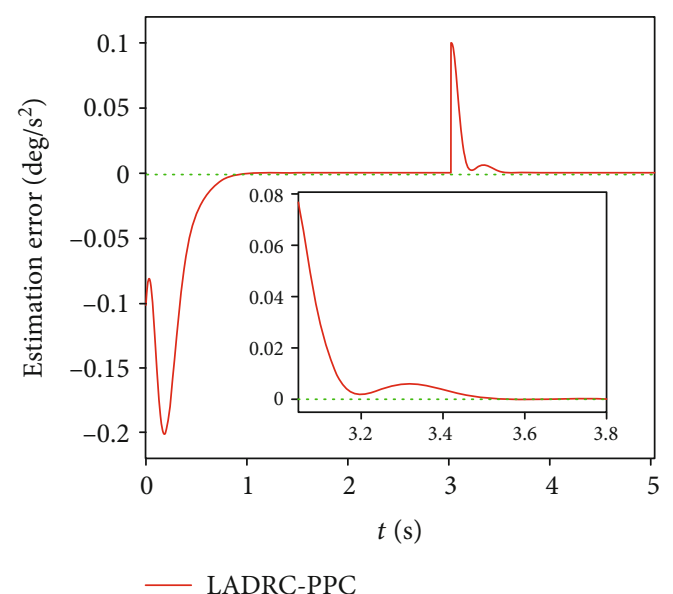

FIgURE 18: Estimation error of $f$ with LADRC-PPC.

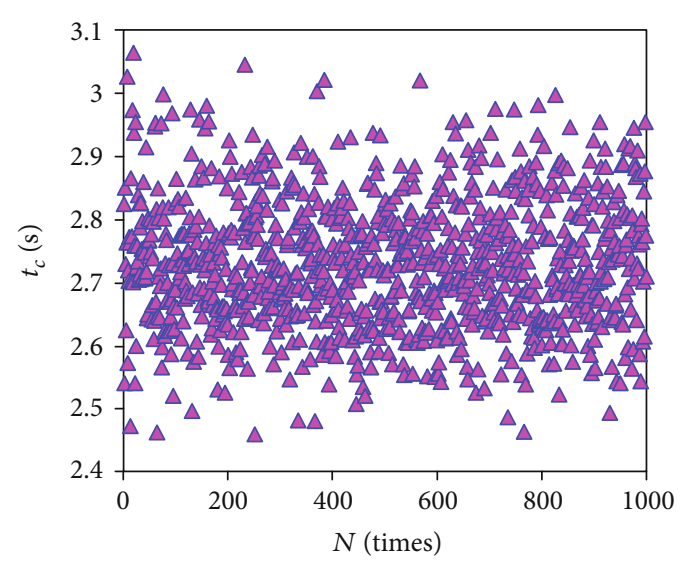

$\triangle$ LADRC

Figure 19: CT of Monte Carlo.

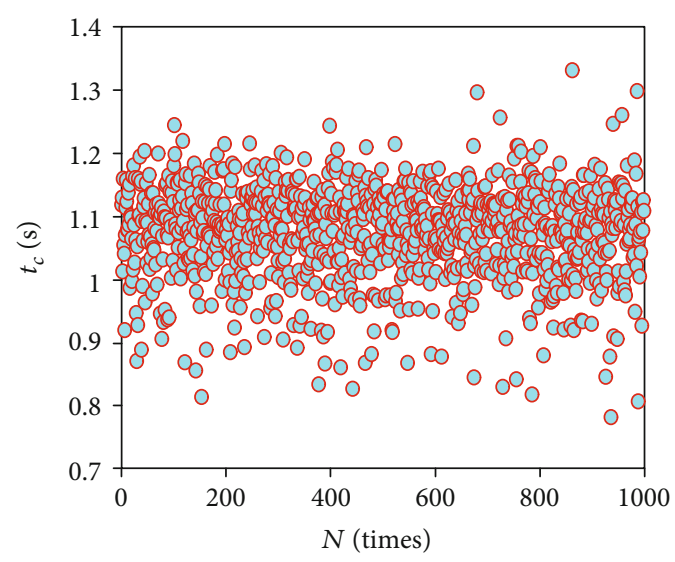

○ LADRC-PPC

Figure 20: CT of Monte Carlo.

of $N=1000$ sample runs is developed, and the parameter perturbations are considered

(1) There is a random variation between $-20 \%$ and $+20 \%$ obeying normal distribution for the aerodynamics coefficients

(2) The perturbation of parameter $J_{z}$ and $\rho$ is $-10 \% \sim$ $+10 \%$ of the standard value, which follows the random variation with normal distribution

Figures 19-24 depicts the 1000-run Monte Carlo simulation results, and Table 3 illustrates the corresponding statistical results including the expectation value $\mu$ and average variance $\sigma$ of the above indexes CT $t_{\mathrm{c}}, \mathrm{AV} \mu_{\mathrm{e}}$, and ACC $Q$.

The Monte Carlo simulation results are depicted in Figures 19-24, and Table 3 lists the statistical results of 1000 Monte Carlo simulations which included the expectation $\mu$ and average variance $\sigma$ of the concerned indexes CT $t_{\mathrm{c}}, \mathrm{AV} \mu_{\mathrm{e}}$, and ACC $Q$. 


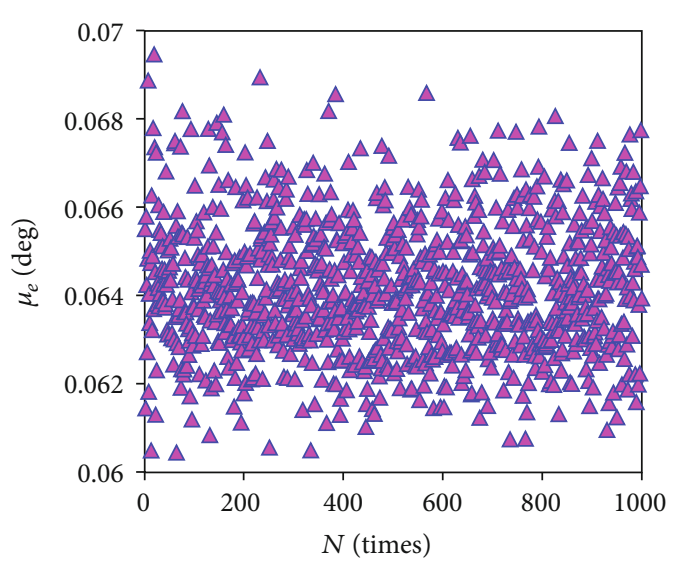

$\triangle$ LADRC

Figure 21: AV of Monte Carlo.

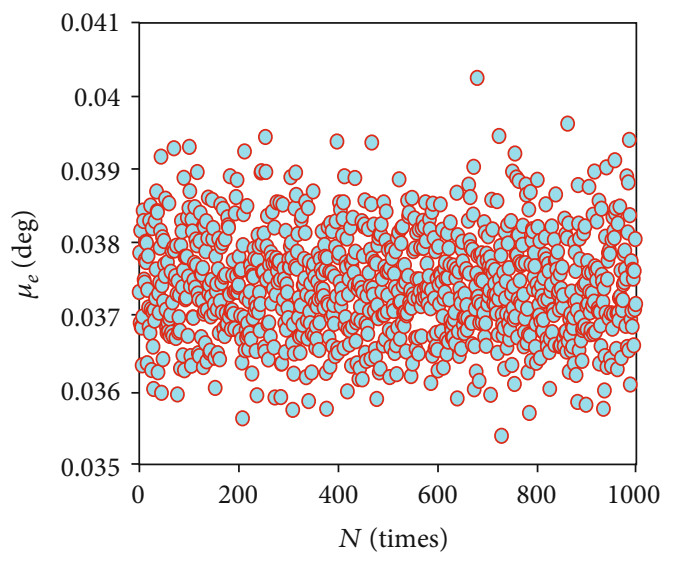

O LADRC-PPC

Figure 22: AV of Monte Carlo.

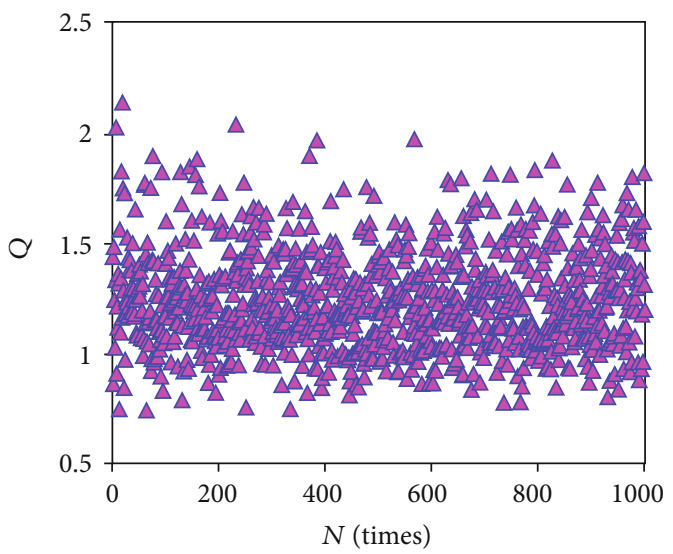

$\triangle$ LADRC

Figure 23: ACC of Monte Carlo.

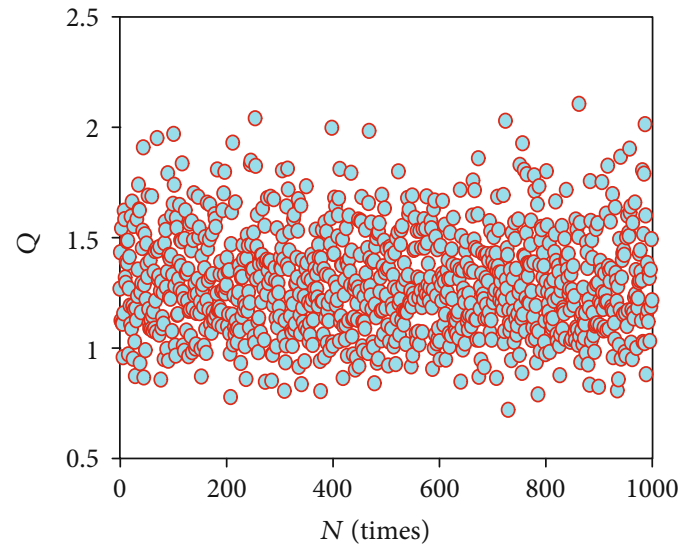

○ LADRC-PPC

Figure 24: ACC of Monte Carlo.

TABLe 3: The statistical results of the Monte Carlo simulations.

\begin{tabular}{lcc}
\hline Statistical data & LADRC & LADRC-PPC \\
\hline$t_{\mathrm{c}}$ & & \\
$\mu$ & 2.7257 & 1.0710 \\
$\sigma$ & 0.1022 & 0.0750 \\
$\mu_{e}$ & & \\
$\mu$ & 0.0641 & 0.0374 \\
$\sigma$ & 0.0015 & 0.0007 \\
$Q$ & & \\
$\mu$ & 1.2270 & 1.2880 \\
$\sigma$ & 0.2253 & 0.2353 \\
\hline
\end{tabular}

Figures 19 and 20 depict the convergence time of the tracking error history for 1000 times with the mentioned two controllers, we can obtain that the expectation of CT $t_{c}$ with the proposed LADRC-PPC $1.07 \mathrm{~s}$ that is shorter than the one of the classic LADRC approach, and it can achieve a smaller dispersion with LADRC-PPC. The statistical results $\mu_{\mathrm{e}}$ exhibit the similar regularity as shown in Figures 21 and 22 . The distributions of the amount of control consumption $Q$ with the above controllers are illustrated in Figures 23 and 24, and the expectation of $Q$ under LADRC-PPC is 1.228 , which is very close to the one of the LADRC method. The above results further verify that the proposed LADRCPPC approach exhibits enhanced robustness despite multiple disturbances, and it can achieve an obviously superior steady-state and transient performances than the classic LADRC-PPC method.

\section{Conclusion}

In this paper, a novel LADRC scheme is proposed for thr ASV attitude control system with multiple disturbances and prescribed performance constraint. The chief feature is that it introduces the PPC technique into the LADRC design 
process so that the tracking error can be strictly confined to an adjustable residual set with the prescribed steady-state and transient performances. Meanwhile, the LESO is employed to estimate the total disturbances such that it can effectively solve the unknown model dynamic problem. Theoretical analysis and contrast simulation results validate that a superior robust tracking performance of the ASV attitude system with the proposed control strategy design. Furthermore, both the transient and steady-state performances of the classical LADRC can be significantly improved by introducing the PPC method. Future work will consider the completely unknown dynamic control system design with actuator saturation via the presented control approach.

\section{Nomenclature}

$\begin{array}{ll}\vartheta: & \text { Pitch angle } \\ \omega_{z}: & \text { Pitch angular rate } \\ \theta: & \text { Flight path angle } \\ \alpha: & \text { Angle of attack } \\ J_{z}: & \text { Pitch moment of inertia } \\ M_{z}: & \text { Pitch moment } \\ S: & \text { Reference area } \\ L: & \text { Reference length } \\ \bar{q}=0.5 \rho V^{2}: & \text { Dynamic pressure } \\ \rho: & \text { Density of air } \\ V: & \text { Velocity } \\ \delta_{z}: & \text { Elevator deflection } \\ \exp (): & \text { Exponential function with natural constant. }\end{array}$

\section{Data Availability}

The data used to support the findings of this study are available from the corresponding author upon request.

\section{Conflicts of Interest}

The authors declared no potential conflicts of interest with respect to the research, authorship, and/or publication of this article.

\section{Acknowledgments}

The authors are grateful for the projects supported by the National Natural Science Foundation of China (Grant No. 11176012).

\section{References}

[1] B. H. Dauby, D. W. Adamczak, J. A. Muse, and M. A. Bolender, "HIFiRE 6: overview and status update 2014," in 20th AIAA International Space Planes and Hypersonic Systems and Technologies Conference, pp. 1-17, Glasgow, Scotland, July 2015.

[2] X. W. Bu, Q. Wang, Y. Zhao, and G. J. He, "Concise neural nonaffine control of air-breathing hypersonic vehicles subject to parametric uncertainties," International Journal of Aerospace Engineering, vol. 2017, pp. 1-16, 2017.

[3] A. Maity and R. Padhi, "Robust control design of an airbreathing engine for a supersonic vehicle using backstepping and UKF," Asian Journal of Control, vol. 19, no. 5, pp. 17101721, 2017.

[4] C. Y. Xu, H. M. Lei, and N. Na, "Active disturbance rejection control for air-breathing hypersonic vehicles based on prescribed performance function," International Journal of Aerospace Engineering, vol. 2019, pp. 1-20, 2019.

[5] D. O. Sigthorsson, P. Jankovsky, A. Serrani, S. Yurkovich, M. A. Bolender, and D. B. Doman, "Robust linear output feedback control of an airbreathing hypersonic vehicle," Journal of Guidance, Control, and Dynamics, vol. 31, no. 4, pp. 10521066, 2008.

[6] G. B. Cai, G. R. Duan, and C. H. Hu, "A velocity-based LPV modeling and control framework for an air-breathing hypersonic vehicle," International Journal of Innovative Computing, Information and Control, vol. 7, no. 5, pp. 2269-2281, 2011.

[7] Z. Cheng, P. Xue, and Y. L. Liu, "Nonlinear controller design for hypersonic vehicles based on dynamic inversion considering flexible states," Advanced Materials Research, vol. 424-425, pp. 701-708, 2012.

[8] G. Wu, X. Meng, and F. Wang, "Improved nonlinear dynamic inversion control for a flexible air-breathing hypersonic vehicle," Aerospace Science and Technology, vol. 78, pp. 734-743, 2018.

[9] G. D. Zhu and Z. J. Shen, "Three dimensional trajectory linearization control for flight of air-breathing hypersonic vehicle," Procedia Engineering, vol. 99, pp. 1108-1119, 2015.

[10] Z. Pu, X. Tan, G. Fan, and J. Yi, "Uncertainty analysis and robust trajectory linearization control of a flexible airbreathing hypersonic vehicle," Acta Astronautica, vol. 101, pp. 16-32, 2014.

[11] X. Cheng, P. Wang, and G. Tang, "Fuzzy-reconstruction-based robust tracking control of an air-breathing hypersonic vehicle," Aerospace Science and Technology, vol. 86, pp. 694-703, 2019.

[12] L. Dou, P. Su, Q. Zong, and Z. Ding, "Fuzzy disturbance observer-based dynamic surface control for air-breathing hypersonic vehicle with variable geometry inlets," IET Control Theory and Applications, vol. 12, no. 1, pp. 10-19, 2018.

[13] A. B. Waseem, Y. Lin, and S. K. Amezquita, "Adaptive dynamic surface control of a hypersonic flight vehicle with magnitude, rate and bandwidth constraints," IFAC Proceedings, vol. 44, no. 1, pp. 5341-5346, 2011.

[14] X. Wang, J. Guo, S. Tang, and S. Qi, "Fixed-time disturbance observer based fixed-time back-stepping control for an airbreathing hypersonic vehicle," ISA Transactions, vol. 88, pp. 233-245, 2019.

[15] X. Bu, G. He, and K. Wang, "Tracking control of air-breathing hypersonic vehicles with non-affine dynamics via improved neural back-stepping design," ISA Transactions, vol. 75, pp. 88-100, 2018.

[16] Y. Yang and Y. Yan, "Neural network approximation-based nonsingular terminal sliding mode control for trajectory tracking of robotic airships," Aerospace Science and Technology, vol. 54, pp. 192-197, 2016.

[17] L. Hu, R. Li, T. Xue, and Y. Liu, "Neuro-adaptive tracking control of a hypersonic flight vehicle with uncertainties using reinforcement synthesis," Neurocomputing, vol. 285, pp. 141-153, 2018.

[18] J. Wang, Q. Zong, R. Su, and B. Tian, “Continuous high order sliding mode controller design for a flexible air- breathing 
hypersonic vehicle," ISA Transactions, vol. 53, no. 3, pp. 690698, 2014.

[19] P. Yu, Y. Shtessel, and C. Edwards, "Continuous higher order sliding mode control with adaptation of air breathing hypersonic missile," International Journal of Adaptive Control and Signal Processing, vol. 30, no. 8-10, pp. 1099-1117, 2016.

[20] Z. Wang, W. Bao, and H. Li, "Second-Order dynamic slidingmode control for nonminimum phase underactuated hypersonic vehicles," IEEE Transactions on Industrial Electronics, vol. 64, no. 4, pp. 3105-3112, 2017.

[21] H. An, C. Wang, and B. Fidan, "Sliding mode disturbance observer-enhanced adaptive control for the air- breathing hypersonic flight vehicle," Acta Astronautica, vol. 139, pp. 111-121, 2017.

[22] R. Parvathy and A. E. Daniel, "A survey on active disturbance rejection control," in 2013 International Mutli-Conference on Automation, Computing, Communication, Control and Compressed Sensing (iMac4s), pp. 330-335, Kottayam, India, March 2013.

[23] H. An, J. Liu, C. Wang, and L. Wu, "Disturbance observerbased antiwindup control for air-breathing hypersonic vehicles," IEEE Transactions on Industrial Electronics, vol. 63, no. 5, pp. 3038-3049, 2016.

[24] Z. Q. Gao, "Scaling and bandwidth-parameterization based controller tuning," in Proceedings of the American Control Conference, pp. 4989-4996, Denver, CO, USA, USA, June 2003.

[25] H. Niu, Q. Gao, Z. Liu, S. Tang, and W. Guan, "Improved linear active disturbance rejection control for lever-type electric erection system with varying loads and low-resolution encoder," Mathematical Problems in Engineering, vol. 2019, 13 pages, 2019.

[26] R. Morales, H. Sira-Ramírez, and J. A. Somolinos, "Linear active disturbance rejection control of the hovercraft vessel model," Ocean Engineering, vol. 96, pp. 100-108, 2015.

[27] L. Zhao, B. Zhang, H. Yang, and Q. Li, "Optimized linear active disturbance rejection control for pneumatic servo systems via least squares support vector machine," Neurocomputing, vol. 242, pp. 178-186, 2017.

[28] S. Ali, G. Yang, and C. Huang, "Performance optimization of linear active disturbance rejection control approach by modified bat inspired algorithm for single area load frequency control concerning high wind power penetration," ISA Transactions, vol. 81, pp. 163-176, 2018.

[29] J. J. Carreño-Zagarra, J. L. Guzmán, J. C. Moreno, and R. Villamizar, "Linear active disturbance rejection control for a raceway photobioreactor," Control Engineering Practice, vol. 85, pp. 271-279, 2019.

[30] H. Li, F. Yu, F. Li, S. Chuang, and Y. Zhide, "Missile attitude control in a large flight envelope based on the linear active disturbance rejection control approach," in Proceedings of 2014 IEEE Chinese Guidance, Navigation and Control Conference, pp. 1219-1223, Yantai, China, August 2014.

[31] H. An and Q. Wu, "Disturbance rejection dynamic inverse control of air-breathing hypersonic vehicles," Acta Astronautica, vol. 151, pp. 348-356, 2018.

[32] J. Na, Y. Huang, X. Wu, G. Gao, G. Herrmann, and J. Z. Jiang, "Active adaptive estimation and control for vehicle suspensions with prescribed performance," IEEE Transactions on Control System Technology, vol. 26, no. 6, pp. 2063-2077, 2018.
[33] Y. Huang, J. Na, X. Wu, and G. Gao, "Approximation-Free control for vehicle active suspensions with hydraulic actuator," IEEE Transactions on Industrial Electronics, vol. 65, no. 9, pp. 7258-7267, 2018.

[34] Z. Zhou, Y. Zhang, and D. Zhou, "Robust prescribed performance tracking control for free-floating space manipulators with kinematic and dynamic uncertainty," Aerospace Science and Technology, vol. 71, pp. 568-579, 2017.

[35] J. Na, Q. Chen, X. Ren, and Y. Guo, “Adaptive prescribed performance motion control of servo mechanisms with friction compensation," IEEE Transactions on Industrial Electronics, vol. 61, no. 1, pp. 486-494, 2014.

[36] S. Wang, J. Na, and X. Ren, "RISE-based asymptotic prescribed performance tracking control of nonlinear servo mechanisms," IEEE Transactions on Systems, Man and Cybernetics: Systems, vol. 48, no. 12, pp. 2359-2370, 2018.

[37] Y. Zhang, S. Wang, B. Chang, and W. H. Wu, "Adaptive constrained backstepping controller with prescribed performance methodology for carrier-based UAV," Aerospace Science and Technology, vol. 92, pp. 55-65, 2019.

[38] C. Ming, R. Sun, and B. Zhu, "Nonlinear fault-tolerant control with prescribed performance for air-breathing supersonic missiles," Journal of Spacecraft and Rockets, vol. 54, no. 5, pp. 1092-1099, 2017.

[39] C. Y. Xu, H. M. Lei, and N. Na, “Active disturbance rejection control for air-breathing hypersonic vehicles based on prescribed performance function," International Journal of Aerospace Engineering, vol. 2018, pp. 1-12, 2018.

[40] C. Zhang, G. Ma, Y. Sun, and C. Li, "Observer-based prescribed performance attitude control for flexible spacecraft with actuator saturation," ISA Transactions, vol. 89, pp. 8495, 2019.

[41] X. Bu, X. Wu, F. Zhu, J. Huang, Z. Ma, and R. Zhang, "Novel prescribed performance neural control of a flexible airbreathing hypersonic vehicle with unknown initial errors," ISA Transactions, vol. 59, pp. 149-159, 2015.

[42] X. Bu, Y. Xiao, and K. Wang, "A prescribed performance control approach guaranteeing small overshoot for air-breathing hypersonic vehicles via neural approximation," Aerospace Science and Technology, vol. 71, pp. 485-498, 2017.

[43] Y. Wang and J. Hu, "Improved prescribed performance control for air-breathing hypersonic vehicles with unknown deadzone input nonlinearity," ISA Transactions, vol. 79, pp. 95107, 2018.

[44] T. Berger, "Tracking with prescribed performance for linear non-minimum phase systems," Automatica, vol. 115, pp. 1$11,2020$.

[45] C. P. Bechlioulis and G. A. Rovithakis, "Robust approximation free prescribed performance control," in Proceedings of the 19th Mediterranean Conference on Control and Automation, pp. 521-526, Corfu, Greece, June 2011.

[46] M. A. Bolender and D. B. Doman, "Nonlinear longitudinal dynamical model of an air-breathing hypersonic vehicle," Journal of Spacecraft and Rockets, vol. 44, no. 2, pp. 374-387, 2007.

[47] J. Parker, A. Serrani, S. Yurkovich, M. Bolender, and D. Doman, "Control-oriented modeling of an air-breathing hypersonic vehicle," Journal of Guidance Control and Dynamics, vol. 30, no. 3, pp. 856-869, 2007.

[48] Q. Zheng, L. Q. Gao, and Z. Q. Gao, “On stability analysis of active disturbance rejection control for nonlinear time- 
varying plants with unknown dynamics," in Proceedings of the 46th IEEE Conference on Decision and Control, pp. 3501-3506, New Orleans, LA, USA, December 2007.

[49] S. Shao and Z. Gao, "On the conditions of exponential stability in active disturbance rejection control based on singular perturbation analysis," International Journal of Control, vol. 90, no. 10, pp. 2085-2097, 2016. 